\title{
ChemComm
}

\section{Monitoring enzymatic ATP hydrolysis by EPR spectroscopy $\dagger$}

Cite this: Chem. Commun., 2014, 50,7262

Received 2nd April 2014

Accepted 7th May 2014

DOI: $10.1039 / c 4 c c 02422 b$

www.rsc.org/chemcomm

An adenosine triphosphate (ATP) analogue modified with two nitroxide radicals is developed and employed to study its enzymatic hydrolysis by electron paramagnetic resonance spectroscopy. For this application, we demonstrate that EPR holds the potential to complement fluorogenic substrate analogues in monitoring enzymatic activity.

Probes that allow directly studying the activity of hydrolytic enzymes have wide-spread applications in biochemistry. ${ }^{1-3}$ In this context, Förster Resonance Energy Transfer (FRET)-based substrate analogues have been extensively used. ${ }^{1-3}$ These probes consist of a cleavage motif for the respective enzyme, e.g. a specific peptide sequence for a protease, which is flanked by two dyes suitable to undergo FRET. ${ }^{4}$ After enzymatic cleavage of the probe, FRET is abolished leading to a large change in the fluorescence characteristics. This concept has been widely applied for studying proteases, ${ }^{1}$ but has also been used for studying various other enzymes. ${ }^{2,3}$

Determination of the distance-dependent dipole-dipole coupling of two electron spins by electron paramagnetic resonance (EPR) spectroscopy potentially offers an alternative way to monitor the cleavage of such analogues (Fig. 1a). In this case, two spin labels (SL) flank the cleavage motif and due to their spatial proximity, dipoledipole coupling occurs in the non-cleaved state. After cleavage, the two spin labels are separated spatially and dipole-dipole coupling vanishes. Depending on the distance distribution of the labels in the intact state, changes in the distance distribution upon cleavage will be detectable by continuous-wave-EPR spectroscopy (cw-EPR, distances $<2.5 \mathrm{~nm})^{5}$ or by double electron-electron resonance spectroscopy (DEER, distances from $1.5 \mathrm{~nm}$ to $10 \mathrm{~nm}$ ) also known as pulsed electron double resonance (PELDOR). ${ }^{6}$

Department of Chemistry and Konstanz Research School Chemical Biology, University of Konstanz, Universitätsstraße 10, 78457 Konstanz, Germany. E-mail:malte.drescher@uni-konstanz.de, andreas.marx@uni-konstanz.de, stephan.hacker@uni-konstanz.de

$\dagger$ Electronic supplementary information (ESI) available: Fig. S1-S4, Table S1, synthesis and characterization of all molecules and detailed experimental procedures. See DOI: $10.1039 / \mathrm{c} 4 \mathrm{cc} 02422 \mathrm{~b}$

\$ These authors contributed equally to this work.

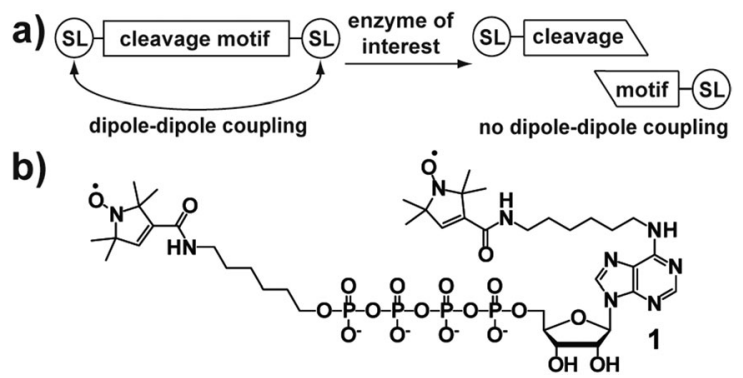

Fig. 1 Concept of the detection of enzymatic activity by EPR. (a) For the envisaged EPR-based technology two spin labels (SL) are attached to a cleavage motif for an enzyme of interest and the change in the dipoledipole coupling after cleavage is monitored. (b) Design of the ATP analogue 1 labelled with two spin labels for studying SVPD activity.

Nitroxide spin labels, which are commonly used in EPR, are less sterically demanding than fluorophores used for FRET applications. ${ }^{7}$ They may therefore cause less interference in the enzymatic activity. Furthermore, EPR allows, in contrast to FRET, detecting the distance distributions over a large range of distances between two identical labels. ${ }^{8}$ It therefore results in a deeper understanding of the actual structure of the probes, which may aid optimizing them. EPR is virtually background free owing to the absence of paramagnetic centres in most biological systems, hence also intracellular measurements are possible. ${ }^{7}$ It also avoids radiation-induced damaging of biological samples due to the long wavelength of the used radiation.

Therefore, it is highly promising to investigate EPR-based probes for their suitability to monitor enzymatic activity. Nevertheless, up to now only the non-enzymatic cleavage of a few very rigid and small molecules has been studied using this approach. ${ }^{9}$ This probe design will not be applicable to typical enzymes. Here, we set out to investigate, whether this approach can also be transferred to large and flexible substrate analogues that could be used to study a larger variety of enzymes. In a benchmark study, we designed and synthesized EPR-based probe 1 (Fig. $1 \mathrm{~b}$ and Scheme S1, ESI $\dagger$ ) to study the activity of nucleotide processing enzymes using an adenosine triphosphate (ATP) core as a cleavage motif and the Phosphodiesterase I extracted from Crotalus adamanteus 


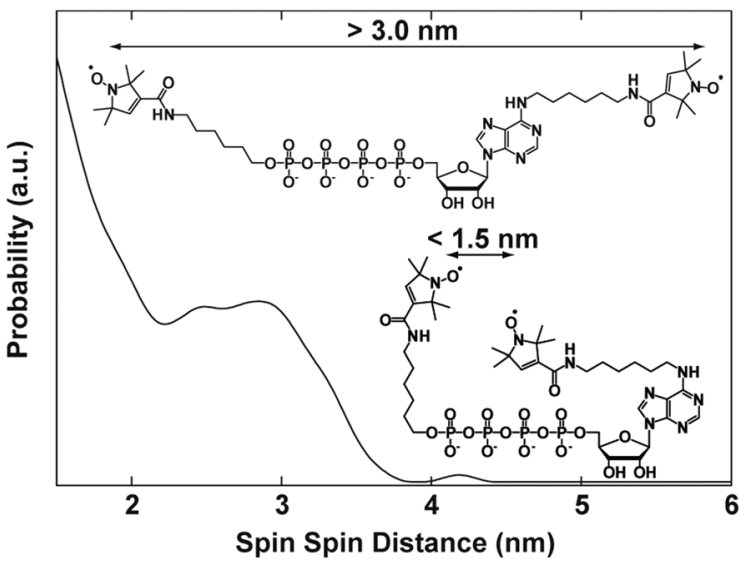

Fig. 2 Distance distribution obtained by model free analysis of the DEER form factor of probe $1 .^{15}$ The inset illustrates the flexibility of probe 1.

(Snake Venom Phosphodiesterase, SVPD) ${ }^{10}$ as model enzyme. Modification of the $\delta$-phosphate of an adenosine tetraphosphate analogue has been chosen as $\delta$-modified tetraphosphate analogues have been shown to be superior substrates to $\gamma$-modified triphosphates for several enzymes ${ }^{11}$ and as phosphoesters have been shown to be stable. ${ }^{12}$ Modification of the N6-position is also well accepted by certain enzymes ${ }^{13}$ and has already been used to study ATP cleavage by SVPD. ${ }^{3}$ Furthermore, we decided to use a nitroxide embedded in a five-membered ring as these labels have been shown to be much more stable than their six-membered counterparts. ${ }^{14}$

To analyse the conformation of probe $\mathbf{1}$ we initially performed DEER to determine the distance distribution between the unpaired electrons (Fig. 2 and Fig. S1, ESI $\dagger$ ) localized on the N-O-bond (pointdipole approximation). It was observed that the two spin labels in probe $\mathbf{1}$ have a broad distance distribution as can be expected due to the very flexible linkers used for their attachment. Furthermore, the distance distribution contains contributions featuring distances around $3 \mathrm{~nm}$, but also a high probability below $2 \mathrm{~nm}$. The larger distances correspond to a largely extended conformation of probe $\mathbf{1}$, whereas the occurrence of short spin-spin distances indicates that probe 1 can also fold in a way that the two labels reside close to each other. In this way, the EPR experiment shows that the flexibility of probes like $\mathbf{1}$ has to be carefully considered, when optimizing their properties. Furthermore, distances shorter than $1.5 \mathrm{~nm}$, which are not accessible with DEER, ${ }^{6}$ seem to occur in a large fraction of the molecules. Therefore, changes in the cw-EPR spectrum of probe 1 should occur during cleavage. ${ }^{5} \mathrm{cw}$-EPR can, in contrast to DEER, be performed using inexpensive easy-to-use equipment and would therefore largely broaden the applicability of an EPR-based approach. ${ }^{16}$

To be able to compare the non-cleaved and the cleaved state of probe 1 by cw-EPR, we next tested, whether SVPD is able to cleave probe 1. For this purpose we incubated probe $\mathbf{1}$ with SVPD in the presence of magnesium chloride. As a negative control the same reaction was set up in the presence of EDTA that inhibits the enzymatic reaction due to complexation of magnesium ions. Analysis by RP-HPLC and HR-MS (Fig. S2, ESI $\dagger$ ) revealed that probe 1 was quantitatively cleaved by SVPD between the $\alpha$ - and the $\beta$-phosphate, whereas no cleavage could be observed in the

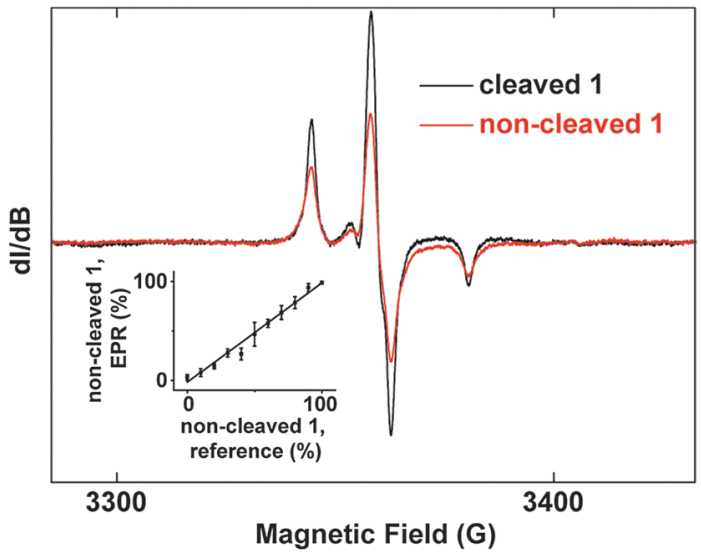

Fig. $3 \mathrm{CW}$-EPR spectra recorded at $-50^{\circ} \mathrm{C}$ in the X-band of probe 1 after incubation with SVPD in the absence (black) or presence (red) of EDTA, normalized to their double integral. Inset: correlation of the known percentage of cleaved probe 1 used in a given CW-EPR experiment (reference) and the percentage of cleaved probe 1 obtained by simulation of the respective EPR spectra. Data points indicate mean \pm standard deviation of triplicates.

presence of EDTA. This shows that probe $\mathbf{1}$ is a substrate for SVPD and might therefore be feasible to monitor its activity.

Next, we measured the X-band cw-EPR spectra of probe $\mathbf{1}$ after incubation with SVPD in the presence or absence of EDTA (Fig. 3). The experiments were set up in the same fashion as described above and after incubation with the enzyme adjusted to a final concentration of $20 \%$ glycerol in order to obtain a glassy solid upon freezing at $-50{ }^{\circ} \mathrm{C}$ as needed for the EPR experiments. In the non-cleaved state a significant broadening of the EPR signal can be detected as compared to the cleaved state. This effect can be attributed to the dipole-dipole coupling of the two labels that are in the range of less than $2 \mathrm{~nm}$ apart from each other. As the cw-EPR spectrum of probe 1 changes during cleavage, it can be used for the concentration-independent detection of the enzymatic activity of SVPD.

To further investigate the applicability of the approach, we mixed different ratios of non-cleaved and cleaved probe 1 to yield a constant total probe concentration and recorded the cw-EPR spectra. The spectra were fitted by a linear combination of the spectra of the completely non-cleaved and completely cleaved state of probe 1 (Fig. S3, ESI $\dagger$ ). Correlating the ratio used in the experiment with the ratio obtained by fitting (Fig. 3) shows that this method can be applied to monitor the fraction of cleaved probe $\mathbf{1}$ in a robust fashion.

Next, we investigated whether the EPR-based approach can also be used to monitor the time-course of the enzymatic reaction of SVPD (Fig. 4). For this purpose, we mixed probe 1, SVPD, magnesium chloride and $20 \%$ glycerol and immediately froze the solution. The initial cw-EPR spectrum was recorded. Afterwards, the solution was warmed to $20^{\circ} \mathrm{C}$ and incubated for the indicated time, before it was frozen again to measure the cw-EPR spectrum of the first time-point. This procedure was repeated for all subsequent time-points. It can be seen that the fraction of non-cleaved probe 1 decreases over time for all enzyme and probe concentrations used and that no cleavage can be detected in the negative control in the absence of SVPD. 


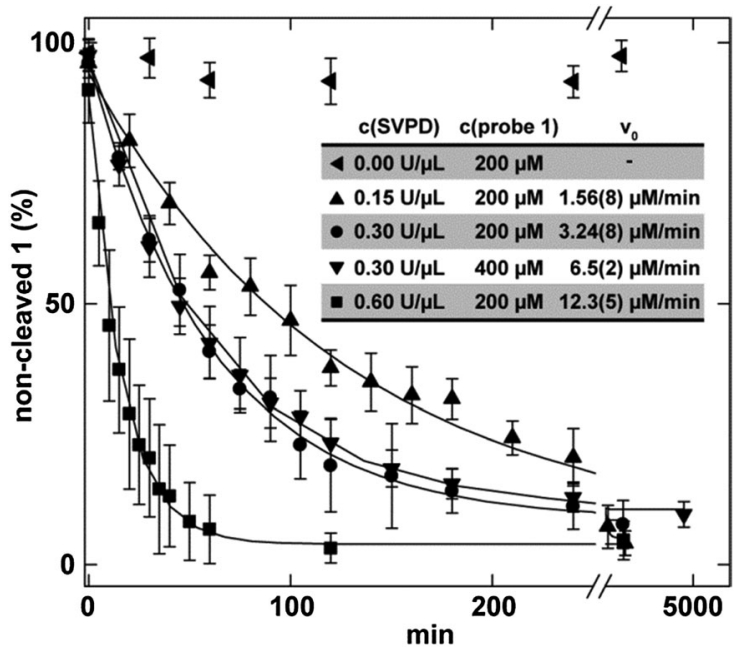

Fig. 4 Time-courses of SVPD reactions monitored by CW-EPR. Data points indicate mean \pm standard deviation of triplicates. Solid lines show exponential fits of the data. These fits were used to calculate the initial velocity $v_{0}$ (see Table S1, ESI, $\uparrow$ for fit parameters).

All curves can be fitted using a monoexponential decay indicating that the used concentration of probe 1 is well below the $K_{\mathrm{M}}$ of the reaction, which is also in line with the fact that doubling the probe concentration leads to doubling of the initial velocity. Correlating the data with the concentration of SVPD used (Fig. 4) shows that the exponential decay time depends linearly on the amount of enzyme for the two lower concentrations used.

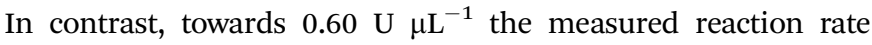
increases more than linearly, probably due to errors introduced by the time elapsing during freezing and thawing. To further exclude that the changes in the cw-EPR spectra are caused by the reduction of one of the two nitroxide radicals, we also monitored the double integral over time (Fig. S4, ESI $\dagger$ ). The double integral is a measure for the number of spins in the sample. It can be seen that the number of spins is constant over time. The change in the EPR spectrum over time is therefore indicative of cleavage of probe 1.

Taken together, we present a novel approach to monitor the activity of hydrolytic enzymes based on the cleavage of large and flexible substrate analogues labelled with two nitroxide radicals. As exemplarily shown for an ATP analogue and SVPD, cleavage of such analogues by the respective enzyme results in vanishing of the dipole-dipole coupling of the two labels. Therefore, the EPR characteristics are changed in a way that allows the robust detection of time-courses of enzymatic cleavage in a concentrationindependent fashion. We are confident that this EPR-based detection of hydrolytic activity will not be limited to monitoring ATP hydrolysis, but will also be applicable to various other enzymes.

Financial support from the DFG (SFB 969) and the Konstanz Research School Chemical Biology is gratefully acknowledged. SMH acknowledges the Konstanz Research School Chemical Biology, the Studienstiftung des deutschen Volkes and the
Zukunftskolleg of the University of Konstanz for a stipend. David Siebert is acknowledged for performing some of the $\mathrm{cw}$ EPR measurements.

\section{Notes and references}

1 (a) E. D. Matayoshi, G. T. Wang, G. A. Krafft and J. Erickson, Science, 1990, 247, 954; (b) P. F. Angelo, A. R. Lima, F. M. Alves, S. I. Blaber, I. A. Scarisbrick, M. Blaber, L. Juliano and M. A. Juliano, J. Biol. Chem., 2006, 281, 3116; (c) B. Korkmaz, S. Attucci, T. Moreau, E. Godat, L. Juliano and F. Gauthier, Am. J. Respir. Cell Mol. Biol., 2004, 30, 801; (d) D. A. Thomas, P. Francis, C. Smith, S. Ratcliffe, N. J. Ede, C. Kay, G. Wayne, S. L. Martin, K. Moore, A. Amour and N. M. Hooper, Proteomics, 2006, 6, 2112; (e) R. Fischer, D. Bächle, M. Fotin-Mleczek, G. Jung, H. Kalbacher and R. Brock, ChemBioChem, 2006, 7, 1428; $(f)$ S. Mizukamia, K. Kikuchia, T. Higuchia, Y. Uranoa, T. Mashimab, T. Tsuruob and T. Naganoa, FEBS Lett., 1999, 453, 356; $(g)$ V. Gurtu, S. R. Kain and G. Zhang, Anal. Biochem., 1997, 251, 98; (h) S. J. Bark and K. M. Hahn, Methods, 2000, 20, 429; (i) Z. B. Packard, V. V. Artym, A. Komoriya and K. M. Yamada, Matrix Biol., 2009, $28,3$.

2 (a) O. Wichmann and C. Schultz, Chem. Commun., 2001, 2500; (b) T. M. Rose and G. D. Prestwich, ACS Chem. Biol., 2006, 1, 83; (c) O. Wichmann, J. Wittbrodt and C. Schultz, Angew. Chem., Int. Ed., 2006, 45, 508; (d) G. Zlokarnik, P. A. Negulescu, T. E. Knapp, L. Mere, N. Burres, L. Feng, M. Whitney, K. Roemer and R. Y. Tsien, Science, 1998, 279, 84; (e) A. Berkessel and R. Riedl, Angew. Chem., Int. Ed. Engl., 1997, 36, 1481; $(f)$ N. Hardt, S. M. Hacker and A. Marx, Org. Biomol. Chem., 2013, 11, 8298.

3 S. M. Hacker, D. Pagliarini, T. Tischer, N. Hardt, D. Schneider, M. Mex, T. U. Mayer, M. Scheffner and A. Marx, Angew. Chem., Int. Ed., 2013, 52, 11916.

4 T. Förster, Ann. Phys., 1948, 437, 55.

5 J. A. Cook and L. J. Brown, Methods Mol. Biol., 2011, 752, 73.

6 (a) G. Jeschke, Annu. Rev. Phys. Chem., 2012, 63, 419; (b) M. Pannier, V. Schädler, M. Schöps, U. Wiesner, G. Jeschke and H. W. Spiess, Macromolecules, 2000, 33, 7812; (c) R. Ward, A. Bowman, E. Sozudogru, H. El-Mkami, T. Owen-Hughes and D. G. Norman, J. Magn. Reson., 2010, 207, 164.

7 M. Azarkh, V. Singh, O. Okle, I. T. Seemann, D. R. Dietrich, J. S. Hartig and M. Drescher, Nat. Protoc., 2013, 8, 131.

8 (a) J. E. Banham, C. M. Baker, S. Ceola, I. J. Day, G. H. Grant, E. J. J. Groenen, C. T. Rodgers, G. Jeschke and C. R. Timmel, J. Magn. Reson., 2008, 191, 202; (b) G. Jeschke, A. Koch, U. Jonas and A. Godt, J. Magn. Reson., 2002, 155, 72; (c) H.-J. Steinhoff, N. Radzwill, W. Thevis, V. Lenz, D. Brandenburg, A. Antson, G. Dodson and A. Wollmeri, Biophys. J., 1997, 73, 3287.

9 (a) P. S. Billone, L. Maretti, V. Maurel and J. C. Scaiano, J. Am. Chem. Soc., 2007, 129, 14150; (b) P. Ionita, A. Volkov, G. Jeschke and V. Chechik, Anal. Chem., 2008, 80, 95.

10 (a) M. A. G. Sillero, O. Madrid, E. Zaera and A. Sillero, Biochim. Biophys. Acta, 1997, 1334, 191; (b) M. Laskowski, Venom Exonuclease, The Enzymes, ed. P. Boyer, Academic Press, 3rd edn, 1971, vol. 4.

11 (a) S. Kumar, et al., Nucleosides, Nucleotides Nucleic Acids, 2005, 24, 401; (b) J. Korlach, et al., Nucleosides, Nucleotides Nucleic Acids, 2008, 27, 1072; (c) J. Eid, et al., Science, 2009, 323, 133; (d) J. Ibach and S. Brakmann, Angew. Chem., Int. Ed., 2009, 48, 4683; (e) B. A. Flusberg, D. R. Webster, J. H. Lee, K. J. Travers, E. C. Olivares, T. A. Clark, J. Korlach and S. W. Turner, Nat. Methods, 2010, 7, 461; $(f)$ A. Sood, S. Kumar, S. Nampalli, J. R. Nelson, J. Macklin and C. W. Fuller, J. Am. Chem. Soc., 2005, 127, 2394.

12 S. M. Hacker, M. Mex and A. Marx, J. Org. Chem., 2012, 77, 10450.

13 S. M. Hacker, N. Hardt, A. Buntru, D. Pagliarini, M. Möckel, T. U. Mayer, M. Scheffner, C. R. Hauck and A. Marx, Chem. Sci., 2013, 4, 1588.

14 M. Azarkh, O. Okle, P. Eyring, D. R. Dietrich and M. Drescher, J. Magn. Reson., 2011, 212, 450.

15 Y. W. Chiang, P. P. Borbat and J. H. Freed, J. Magn. Reson., 2005, 172, 279.

16 Y.-W. Chiang, T.-Y. Zheng, C.-J. Kao and J.-C. Horng, Biophys. J., 2009, 97, 930. 